\section{$(\sqrt{1})>$ \\ HORIZONTES}

www.revistahorizontes.org
Horizontes. Revista de Investigación en Ciencias de la Educación Https://doi.org/10.33996/revistahorizontes.v4i15.111

Julio - Septiembre 2020

Volumen 4 / No. 15

ISSN: $2616-7964$

ISSN-L: $2616-7964$ pp. 234 - 245

\title{
Educación emocional y su incidencia en el aprendizaje de Ciencias Naturales en niños con TDAH
}

\author{
Emotional education and its incidence in the learning of Natural Sciences in children \\ with ADHD
}

\section{Educação emocional e sua incidência na aprendizagem das Ciências Naturais em crianças com TDAH}

\section{ARTÍCULO ORIGINAL}

Gustavo Eleodoro Valverde Peralta gusvalverdep92@gmail.com

ORCID: 0000-0002-4512-5857

Mayko Carlos Sumba Bautista maykosumba@gmail.com

ORCID: 0000-0001-5588-9685
Elka Jennifer Almeida Monge ealmeidam@unemi.edu.ec ORCID: 0000-0001-8507-5949

María Gabriela Jiménez Vilema gabriela14abigail@gmail.com

ORCID: 0000-0002-4101-2484

Universidad Estatal de Milagro, Ecuador

Recibido abril 2020 | Revisado mayo 2020 | Publicado en julio 2020

\section{RESUMEN}

El presente artículo de investigación tiene como objetivo mostrar un análisis de la relación que existe entre la educación emocional en el aprendizaje de las ciencias naturales en los alumnos con TDAH. En este estudio se evaluó el factor emocional, como el pilar esencial para un óptimo aprendizaje. Se seleccionó a una muestra específica de niños cuyo diagnóstico sea de TDAH, obteniendo una cantidad de 50 , procedentes de cursos básicos de las instituciones educativas de primaria. Se aplicó el TMMS24, para medir sus emociones en relación a las calificaciones de los niños, y los registros de notas para verificar su aprendizaje en la asignatura específica. Mediante un análisis estadístico del SPSS V.25 y a través de la técnica de correlación de Pearson se encontró una relación altamente significativa entre los factores emocionales y el desarrollo del aprendizaje en la asignatura de ciencias naturales. Con estos resultados se observa que existe una respuesta emocional favorable en niños con TDAH y se concluye que el logro de aprendizaje es significativo en la asignatura de ciencias naturales.

Palabras clave: Aprendizaje; ciencias naturales; educación; emociones, TDAH

\begin{abstract}
This research article aims to show an analysis of the relationship between emotional education in the learning of natural sciences in students with ADHD. In this study the emotional factor was evaluated as the essential pillar for optimal learning. We selected a specific sample of children whose diagnosis is ADHD, obtaining a number of 50 , from basic courses of primary schools. The TMMS24 was applied to measure their emotions in relation to the children's grades, and the grade records to verify their learning in the specific subject. Through a statistical analysis of SPSS V.25 and through Pearson's correlation technique, a highly significant relationship was found between emotional factors and the development of learning in the natural science subject. With these results it is observed that there is a favorable emotional response in children with ADHD and it is concluded that learning achievement is significant in the subject of natural sciences.
\end{abstract}

Key words: Learning; natural sciences; education; emotions, ADHD 


\section{RESUMO}

0 presente artigo de pesquisa tem como objetivo apresentar uma análise da relação que existe entre a educação emocional na aprendizagem das ciências naturais em alunos com TDAH. Neste estudo, o fator emocional foi avaliado como o pilar essencial para 0 aprendizado ideal. Foi selecionada uma amostra específica de crianças com diagnóstico de TDAH, obtendo-se um número de 50 , em cursos básicos de instituições de ensino fundamental. O TMMS24 foi aplicado para mensurar suas emoções em relação às notas das crianças e os registros de notas para verificar sua aprendizagem na disciplina específica. Por meio da análise estatística do SPSS V.25 e da técnica de correlação de Pearson, foi encontrada uma relação altamente significativa entre os fatores emocionais e o desenvolvimento da aprendizagem na disciplina de ciências naturais. Com esses resultados, observa-se que existe uma resposta emocional favorável em crianças com TDAH e conclui-se que o aproveitamento escolar é significativo na disciplina de ciências naturais.

Palavras-chave: Aprendizagem; ciências naturais; educação; emoções, TDAH

\section{INTRODUCCIÓN}

El estudio de las emociones en el contexto educativo ha crecido constantemente, debido al interés por investigadores en relación a las diversas problemáticas que surgen a raíz de este componente sustancial. Las emociones vinculadas a la educación forman una sola fuerza que impulsa y contribuye en el logro de sus objetivos académicos en los estudiantes, sobre todo en aquellos que inicia su etapa de escolarización, donde el apoyo de sus padres es una pieza clave para su correcto desempeño. Sin embargo, en los tiempos actuales la familia ha dejado de tener protagonismo en el desarrollo escolar de los hijos, dado las múltiples causas como un desinterés por su responsabilidad con su progenitor, conocido como negligencia, la falta de paciencia para trabajar conjunto con el hijo las actividades de la escuela, o en ocasiones los padres tienen ocupaciones que no da el espacio necesario para compartir escuela con ellos. A esto también se suma la falta de empatía en los docentes y también se incluye la mala pedagogía que muchas veces se evidencia en el resultado de aprendizaje de los niños. De esta forma es como se puede apreciar que a largo plazo existen consecuencias puesto que la ausencia de apoyo desvirtúa todo objetivo que un estudiante se plantea en su contexto educativo.

El presente estudio científico busca determinar como la educación emocional influye en el logro de aprendizaje de los niños en la asignatura de ciencias naturales, la investigación enfatiza su análisis en los niños que presentan problemas relacionados al TDAH, ya que aquellos infantes que tienen diagnóstico presentan dificultades para poder mantener un equilibrio emocional, además de que en contexto de clases su condición no se compagina donde regularmente los niños aprenden, es decir que sus alteraciones hacen que se desinterese por el aprendizaje y genere malestar durante la jornada académica. El análisis de los factores vinculados a las emociones permite percibir el grado de aceptación que tienen los niños con la asignatura elegida para determinar la presencia del problema.

Las emociones son representaciones biológicas y cognitivas, que se dan forma en el área social. Si las emociones, tienen como consecuencia sentimientos de satisfacción y placer en un individuo, se consideran emociones positivas; sin embargo, en el caso opuesto, dónde tenemos percepciones de inconformidad o una situación que afecte la salud mental, sean irritabilidad ansiedad, temor, entre otros, son consideradas emociones negativas. Las emociones tienen manifestaciones variadas en su energía, pueden ser altas o bajas en sus expresiones, sin duda, y también pueden interactuar en un individuo, una variedad de emociones en su clímax al mismo tiempo. (Aires et al., 2006) 


\section{MÉTODO}

El estudio fue de análisis cuantitativo, esta modalidad permite percibir un problema partiendo de resultados obtenidos. Se considera un diseño no experimental, debido que no existe manipulación de las variables educación emocional y aprendizaje, y de corte transversal, ya que se realizó la evaluación en el campo específico, es decir en las aulas de clases, en una sola instancia, por lo que, se pudo apreciar los resultados en tiempo real. Los análisis que se emplearon fueron el descriptivo, cuyo propósito está en el detalle de los resultados relacionados a los criterios emocionales y el promedio de calificaciones de a asignatura, el explicativo que da razón a los datos obtenidos producto de una interpretación de los factores emocionales y el aprendizaje de la asignatura. Y correlacional, este último método específico permitió analizar la relación directa entre los factores (atención, claridad, regulación y aprendizaje).

En cuanto a los participantes, se consideró que los mismos fueran de instituciones educativas públicas, donde mayormente se pueden observar este tipo de situaciones, para el estudio respectivo se seleccionó una muestra representativa de 50 niños cuyos diagnósticos son de TDAH. En lo referente a la forma de evaluación, se ha elegido el cuestionario TMMS24 (Fernández y Extremera 2005), conocido también como Trait Meta- Mood Scale, tiene sus bases en estudios de Myer, Salovey y Goldman (1995), consta de 24 ítems, con alternativas múltiples, este instrumento tiene como objetivo medir la reacción emocional en los niños, de la misma forma los factores que lo integran son: atención, claridad y regulación. En cuanto a su fiabilidad, fue analizado mediante la prueba de KMO, donde se obtuvo una puntuación entre 0,772 y 0,867 lo que deja en evidencia que en el análisis factorial es viable su aplicación, de la misma forma mediante el método Alfa de Cronbach, sus cifras oscilan entre 0.85 y 0.88 ( Ángulo y Albarracín , 2018).

En tanto que, para la otra variable de aprendizaje, fue necesario solicitar los reportes de calificaciones de las asignaturas en la institución, mismas que son: matemáticas, estudios sociales, lenguaje y finalmente ciencias naturales, que es la asignatura de interés de este estudio. La información fue procesada en el programa SPSS vs. 25 donde se establecieron los respectivos análisis estadísticos para poder corroborar el objetivo de la investigación.

\section{RESULTADOS}

Los valores reflejados en la tabla 1 , mostraron los estadísticos descriptivos de la variable edad en los niños, donde la edad fue representada por la media de los participantes 7.8, la cual indicó el promedio de evaluados, cuyas edades consideradas para el estudio fueron de 7 a 10 años, no obstante se encontró una mediana de 8 , que se denominó la edad central debido a la gran frecuencia en la población y la moda se determinó en 9, indicando que la mayoría de los encuestados poseían dicha edad, los demás entre 7,8 y 10 años. La desviación estándar fue de ,653 de las edades promedio y una varianza de ,426, las cuales representaron la distancia entre edades. 
Tabla 1. Análisis descriptivo de las variables sexo y edad

\begin{tabular}{lr} 
Media & Edad \\
Mediana & 7.8 \\
Moda & 8 \\
Desviación estándar & 9 \\
Varianza &, 653 \\
\hline
\end{tabular}

Base: estudiantes de institución educativa

Posteriormente, en la tabla 2 se muestra que el problema relacionado al TDAH en la muestra seleccionada, fue más recurrente en niños que en niñas, lo que evidencia que el problema del trastorno recae en la población masculina.

Tabla 2. Análisis porcentual de incidencia del problema en género

\begin{tabular}{llcccc} 
& & Frecuencia & Porcentaje & Porcentaje válido & $\begin{array}{r}\text { Porcentaje } \\
\text { acumulado }\end{array}$ \\
Válido & Masculino & 38 & 76,0 & 76,0 & 76,0 \\
& Femenino & 12 & 24,0 & 24,0 & 100,0 \\
& Total & 50 & 100,0 & 100,0 & \\
\hline
\end{tabular}

Base: estudiantes de institución educativa

Seguido en la Tabla 3 se muestra el análisis descriptivo de las emociones, relacionado a las actitudes que tienen los niños en relación a sus emociones, y según manifestó el resultado obtenido el $58 \%$ responden a problemas en relación a la regulación de sus emociones, con un $24 \%$ en lo que respecta a su claridad o comprensión, y el $18 \%$ en lo referente a la atención, cabe destacar que estas son las posturas emocionales que los niños adoptan frente a las exigencias de la asignatura. Las actitudes emocionales que los niños adopten por el aprendizaje son muy sustanciales debido que las emociones constituyen un motor esencial para la adquisición de conocimientos y una mejor comprensión del aprendizaje en sus diversas etapas de escolarización, la atención que destinen para poder captar los conceptos, la claridad con la que se comprenden, y la regulación de las intensidades emocionales para poder responder de forma oportuna los diversos desafíos. 
Tabla 3. Análisis descriptivo emocional

\begin{tabular}{llcccc} 
Válido & Frecuencia & Porcentaje & Porcentaje válido & $\begin{array}{r}\text { Porcentaje } \\
\text { acumulado }\end{array}$ \\
$\begin{array}{l}\text { Atención } \\
\text { emocional } \\
\text { Claridad } \\
\text { emocional }\end{array}$ & 11 & 18,0 & 18,0 & 22,0 \\
& 17 & 24,0 & 24,0 & 62,0 \\
$\begin{array}{l}\text { Regulación } \\
\text { emocional }\end{array}$ & 22 & 58,0 & 58,0 & 94,0 \\
Total & 50 & 100,0 & 100,0 & \\
\hline
\end{tabular}

Base: estudiantes de institución educativa

Se observó con detalle el porcentaje de rendimiento de los estudiantes al respecto de la asignatura de ciencias naturales, donde el 36\% de promedio es regular y bueno, en tanto que el 9\% refleja insuficiencia y el 5\% de ellos son excelentes en la Tabla 4. Estos resultados fueron procesados con la finalidad de poder apreciar el nivel de desempeño en cuanto a calificaciones según su cumplimiento $\mathrm{y}$ participación, así como evaluación de la materia. En los registros de calificaciones se puede apreciar que el rendimiento promedio es moderadamente aceptable, dado los resultados de su actividad dentro de las aulas de clases, con lo que es más factible relacionarlo con sus estadios emocionales según los resultados obtenidos de la prueba aplicada.

Tabla 4. Análisis descriptivo de calificaciones de asignatura de Ciencias Naturales

\begin{tabular}{llcccc} 
Válido & Frecuencia & Porcentaje & Porcentaje válido & $\begin{array}{r}\text { Porcentaje } \\
\text { acumulado }\end{array}$ \\
& Insuficiente & 9 & 18,0 & 18,0 & 18,0 \\
& Regular & 18 & 36,0 & 36,0 & 54,0 \\
& Bueno & 18 & 36,0 & 36,0 & 90,0 \\
& Excelente & 5 & 10,0 & 10,0 & 100,0 \\
& Total & 50 & 100,0 & 100,0 & \\
\hline
\end{tabular}

Base: estudiantes de institución educativa

En la Tabla 5 se representó el análisis de correlación de Pearson, donde se ha puesto en evidencia los factores del instrumento TMMS24 y los registros de calificaciones de los estudiantes, los resultados indican que en la asignatura de Ciencias Naturales, el índice de correlación es significante y significativa con relación a la atención emocional $(\mathrm{p}=071)$, con respecto a la claridad emocional $\left(\mathrm{p}=109^{*}\right)$ y en la regulación emocional $\left(\mathrm{p}=265^{* *}\right)$, todos los valores descritos bajo el sig. bilateral de (003; 003; 001<005) con lo que se pudo indicar que existió conexión entre la actitud emocional de los niños en relación a la materia, a diferencia de las demás donde los resultados son menores.

El índice de relación entre los factores desarrollados fue significativo, por lo cual existe una reacción emocional en atención a la asignatura de Ciencias Naturales, no obstante, las demás 
materias guardan una relación discreta, lo que pone en evidencia que los niños se sienten atraídos por la asignatura de Ciencias Naturales de estudio en particular.

Tabla 5. Análisis correlacional

\begin{tabular}{llcccc} 
& & Matemáticas & $\begin{array}{c}\text { Ciencias } \\
\text { Naturales }\end{array}$ & $\begin{array}{c}\text { Estudios } \\
\text { Sociales }\end{array}$ & Lenguaje \\
Atención emocional & $\begin{array}{l}\text { Correlación de } \\
\text { Pearson }\end{array}$ &, $135^{*}$ &, $071^{*}$ &, $335^{*}$ &, $205^{*}$ \\
& Sig. (bilateral) &, 005 &, 003 &, 005 &, 002 \\
& $\mathrm{~N}$ & 50 & 50 & 50 & 50 \\
Claridad emocional & Correlación de &, $092^{*}$ &, $109^{*}$ &, $015^{* *}$ &, $057^{*}$ \\
& $\begin{array}{l}\text { Pearson } \\
\text { Sig. (bilateral) }\end{array}$ &, 004 &, 003 &, 000 &, 002 \\
& N & 50 & 50 & 50 & 50 \\
Regulación emocional & Correlación de &, $143^{*}$ &, $265^{* *}$ &, $088^{*}$ &, $005^{*}$ \\
& $\begin{array}{l}\text { Pearson } \\
\text { Sig. (bilateral) }\end{array}$ &, 002 &, 001 &, 005 &, 001 \\
& N & 50 & 50 & 50 & 50 \\
\hline
\end{tabular}

**. La correlación es significativa en el nivel 0,05 (bilateral).

Base: estudiantes de institución educativa

\section{Discusión}

Las emociones tienen respuesta a los estímulos que producen un agente detonante, en el que exista una reacción, cada uno desencadena respuestas distintas. Los tipos de respuesta o emociones, pueden ser interno o externo, asociadas a una situación de dependencia social, otras que son por impulsos viscerales, y otras que superpuestas en base a las primarias que cambian las primeras como consecuencias de estas (Bermeo et al., 2012). Que por mencionar algunos tipos de emociones como ira, alegría, tristeza, miedo que producen las emociones positivas, negativas. Todas estas mencionadas tienen factores subyacentes que ligan unas a otras. El reconocimiento de las emociones en los resultados ha sido evidenciado a través de los factores atención, claridad y regulación emocional, cada uno constituido por una reacción ante el estímulo académico, en el caso de la asignatura de ciencias naturales.

La inteligencia emocional permite tener el conocimiento para basar la psicología actual. De este enfoque que se encarga del estudio de los trastornos mentales y la capacidad de razonamiento, y se llega a la comprensión que las emociones son internas en relación al comportamiento, que no son patología y qué se pueden aprender si se profundiza en el estudio como es el ser humano realmente.

Ciertos estudios demuestran lo contrario, en sustento a la parte académica, proponiendo que las emociones son indistintas al momento de determinar la capacidad intelectual en los estudiantes (Blanco, de Caso, y Navas, 2013). 
Una réplica que flota es que los jovenes que tienen una inteligencia emocional adecuada tienen bienestar en su salud mental, y reubica la relevancia de la emocion en la educación como estudio considerable llevandola a un plano no solo estudiantil sino otros aspectos como la vida laboral, familiar, e interacciones sociales en general (Bustillo y Servera, 2015).

Esta rama de la educación que ayuda a fortalecer habilidades y destrezas del ser humano en su parte emocional. El individuo se encuentra en un mundo donde se sortean experiencias y eventos, que necesitan el manejo adecuado de su capacidad de reacción para tener control y empoderamiento, para tener el equilibrio en dichas situaciones. La educación emocional ha tenido que actualizar su concepto con el paso del tiempo, para ajustarse a la realidad que el manejo de las emociones amerite, en la sociedad cambiante que se vive (Bravo, 2018). Se puede apreciar en los resultados que en las otras asignaturas la relación es normal estadísticamente, esto indica que existe una mejor respuesta emocional por parte de los niños.

La relación entre la educación emocional y la inteligencia emocional es estrecha, pues se busca que la primera llega a la segunda en la práctica del yo, que cada individuo tiene con su parte cognitiva para manejar de forma óptima sus emociones. La experticia a través de la crítica ayuda a la educación emocional a manejar de buena manera las emociones, pero hace falta captar mucho las percepciones de los sentidos, sin duda (Borrachero et al., 2014). En los resultados se puede apreciar la relación de la reacción emocional de los estudiantes frente a sus asignaturas, donde se establece una significancia entre las emociones y el aprendizaje.

Algunos resultados de estudios realizados en niños con TDAH, demuestran tiene poco reconocimiento de emociones, y de emociones diversas como el miedo, la ira, se encuentra la probabilidad de que en esta población sea mayor el déficit. El objetivo del estudio es el direccionamiento al diagnóstico educativo y clínico de los niños con TDAH, y así buscar estrategias para implementar, y mejorar el rendimiento académico. Ajustar adaptaciones curriculares en que se contemple el manejo de la Inteligencia Emocional que serían un gran apoyo en la educación de estos niños (Orozco y Zuluaga, 2015).

Muchos autores destacan el beneficio que la educación emocional ha tenido en el niño en su desarrollo educativo. Hay En programas con desarrollo integral en los niños, demostrando que existen sectores vulnerables que mejoran significativamente en la escolaridad cuando se satisfacen necesidades básicas, es importante entender cómo ayudar a los alumnos para lograr el alcance deseado (Acero, 2013).

La expresión de las emociones es la capacidad de manera eterna demostrar lo que sucede en lo interior al procesar la información que se recibe. Los niños que no pueden expresarse, suelen ser catalogados de rebeldes, de muchas maneras (Lolas, 2015). Tienden a estar socialmente distantes, por no poder extender su lenguaje emocional, de lo que siente, pero no puede expresarlo, provocan reacciones adversas de los otros.

Los niños con TDAH, se encuentran propensos a la frustración, aislamiento social, depresión, soledad, y dificultades académicas. Hay que considerar que estos niños dependen que una atención especial integral a sus necesidades para favorecer su desempeño estudiantil. Hay que evitar en lo posible ambientes que aumenten las probabilidades de generar ansiedad, confusión y malestar en el aprendizaje, los test de sensibilidad no verbal infantil, para tener en cuenta que sus emociones están de acuerdo con sus capacidades cognitivas (Yadira, 2012). En los resultados se 
puede señalar que existen dificultades en las respuestas o actitudes emocionales en función de su aprendizaje. Las personas con TDAH, tiene un serio inconveniente con la empatía, no tienen una comprensión clara de las sensaciones de las otras personas. La dificultad para generar relaciones sociales, les cuesta establecer estrategias para generar pronósticos a los resultados de ciertas conductas, suelen ser solitarios en los grupos sociales de los cuales es por el rechazo que su propia conducta provoca (Luzuriaga, 2018).

En la actualidad las emociones cumplen un rol importante en el área del aprendizaje, debido que se las usa con emociones positivas en la enseñanza, pueden mejorar la memoria y aprendizaje del individuo, así lo que no era de interés o de su gusto va a quedar en la mente de la persona. Se estima, por lo tanto, que las emociones y aprendizaje van de la mano, pues se ha mejorado la parte cognitiva en el aprendizaje, se las han utilizado en las orientaciones modernas (Marcos, 2019).

Los niños que tienen cuadro clínico de Trastorno por Déficit de Atención e Hiperactividad, (TDAH), se muestran negativas al aprendizaje. Suelen tener frustración por su falta de desarrollo académico, tiene problemas familiares por sus estudios, requieren apoyo, en conjunto inconvenientes constantes en estos ambientes (Burrola, Burrola y Viramontes, 2016)__Necesitan una motivación en las áreas educativas en las cuales deban desenvolverse, pues si reciben esa contemplación que sería adecuada para su condición, sentirán comodidad para llevarlas a cabo, sino se presenta esto sentirán dificultad para concentrarse y su atención académica se disminuirá hasta ser una actividad tediosa la cual será difícil que la lleven a cabo.

Las personas que presentan TDAH, tienen poco control de las emociones, con una deficiencia a nivel neurológico de empatía porque sus estadios emocionales se presentan de manera intensa, que se vuelven para ellos incontrolables, y su atención desencadena desubicación. Manejar sus emociones para encontrar el equilibrio es un reto, en esta parte es donde se propone el aporte significativo que brinda la educación emocional (Reche, 2016).

La variabilidad de los estudiantes con TDAH, es considearada cambiantes en niveles bajos y altos de expresión, y otros en estado opuesto como insensiblidad aparente, apatía cruda, debido a su poca capacidad en la regulación de sus emociones. Entonces se puede decir, que su exterioración de las emociones, se dificulta al igual que la comunicación, entre los que se pueden observar epxresiones motoras o verbales con cierto desorden en el contexto, provocando estrés, ansiedad o depresión (Yadira, 2012).

Las competencias emocionales en el área educacional han aportado de manera considerable. Cada individuo asociado a su propia competencia emocional y motivada en su mundo, se podría decir, he demostrado un mayor rendimiento académico, por el tutor que ha logrado llegar al esquema de emociones lo mayor cercano posible (Santurde y Del Barrio , 2013). Entonces se puede apreciar la competencia emocional de cada estudiante, la capacidad de llegar a la competencia emocional, el proceso educativo y el rendimiento educativo como resultado.

Considerando el área de educación infantil en el contexto de este estudio, se manejan los objetivos contemplados, entre estos tenemos, mejorar el desarrollo integral del niño/a, desarrollar actitudes que tengan que ver a los valores en la formación, por igual forma en potencializar las actitudes en la satisfacción del deber cumplido, tener la capacidad de tolerar situaciones que generen estrés, evitando la desesperación y tomando acciones correctas. 
Reconocer las propias emociones que surgen ante un evento y lo que se puede y lo que no se puede hacer, se conoce como la capacidad de inteligencia Emocional, para encontrar el discernimiento de uno mismo. La voluntad de encontrarse en los sentimientos de manera intrapersonal. Las personas que tiene Inteligencia emocional, logran dirigir su vida y mejorar ante situaciones adversas, conseguir metas aportando seguridad en su propia persona, no es que tengan menos problemas, pero si la capacidad de poder manejarla (González, Bakker, y Rubiales, 2014). En las personas que tienen TDAH, si se logran mejorar la expresión de sus sentimientos podrían tener u buen desempeño académico, social y por ende una buena calidad de vida.

En diferentes estudios de la Inteligencia Emocional, si una persona está consciente de su estado de ánimo y de su respuesta a los pensamientos que subsiguen. Así tiene una consciencia claro sobre sí mismo, sabe con precisión su reacción evitado una acción inadecuada (Vásquez, 2017). Lo que en el caso de los niños con TDAH es un tema complejo de manejar, dada su dificultad para sobrellevar situaciones que rápidamente le generan estrés y exaltación emocional.

La perspectiva propia en reconocer nuestras emociones y la reacción en conducta que produce es determinante cuando estas aparecen. Tenemos situaciones que no apreciamos, por no saber, si tenemos una respuesta negativa debido a que no hay conciencia en dicha reacción. Sin embargo, en el momento que se educa el individuo, puede analizar y procesar dicha información emocional, para regular su conducta de manera que sea prudente al contexto (Fernández, et al., 2011). La autoevaluación es crucial y permite cambiar las sensaciones negativas, creando perspectivas mejores sobre el mismo asunto (Aires et al., 2006). La estructura de la
Inteligencia Emocional, es manejar esas emociones por positivas a futuro según se interioriza. Por ejemplo, si alguien charla con una persona que le desagrada tendrá sensaciones negativas, pero si maneja sus emociones, cambiará la emoción por sensaciones positivas.

Las ciencias naturales ayudan a entender el mundo que rodea en su complejidad, permite desarrollar capacidades intelectuales, generando estrategias para poder operar en la realidad, conociéndola y reformándola, dando paso a la ciencia en su estudio para bien común. Con sus variables en el tiempo debido a los avances que se producen en su desarrollo. El facilitador de esas percepciones, debe tener argumentos estratégicos para la comprensión de sus enseñados, transmitirlos correctamente con creatividad pedagógica (Tacca Huamán, 2010).

La idea es generar amplitud cognitiva, los fenómenos comprendidos siempre proyecta otros eventos cuestionables pues la ciencia no es finita. Los paradigmas pueden reformularse, la ciencia puede ser enseñable, puede ser aprendida en el contexto buscando el tutor su correcta manera de llegar a sus alumnos. La reflexión y los cuestionamientos vivos es lo que produce, en pro de su expansión. La ciencia no se detiene en el tiempo.

En los niños con TDAH, la atención es un tema recurrente por su híper focalización, es un reto para cualquier facilitador de la educación, lo cual buscar estrategias para mejorar esa conducta sería apropiado. Tener la libertad de explorar en un ambiente controlado es una opción para ellos. Las ciencias naturales aportan mucho a la parte de la experimentación, sin recurrir tanto a las teorías $\mathrm{y}$ palabras que duran mucho tiempo que provocan ansiedad en niños con TDAH (Alfaro, 2012). Los ambientes de estudio en esta asignatura son variados e interminables en su 
saber. Las probabilidades de tener un niño con TDAH, apático resultan proporcionales al tutor que dirige la estrategia pedagógica (Ozdemir, 2010).

El método de aprendizaje ERCA, basado en las técnicas de Piaget y David Kolb (1984), es un ciclo de aprendizaje en el que tiene como objetivo, generar conocimiento de experiencias concretas sujetas a nuevas experiencias, tenemos capacidades de: experiencia concreta, observación-reflexión, conceptualización, experimentación. Estos ejes pueden ser alternados por el enseñado, en cualquiera de sus etapas, lo que se conoce como el cirulo de aprendizaje. Debido a que cada sujeto tiene sus competencias para desarrollar conocimiento, la idea es que los preconceptos sean capaces de evidenciarse o reformularse en la medida que la experiencia aporte a la nutrición de respuestas y puedan así derribar prejuicios (Kolb, 1984).

El método ERCA, con su ciclo de aprendizaje: la experiencia, la observación y reflexión, la conceptualización y la aplicación. En un principio practico, se explorar las bases de algo, se actúa, se hace y explora. Todos los procesos son supervisados tanto en la práctica como en la operación (Scandar, 2013). Es la base para partir a la experiencia a la educación. Para lograr en aprendizaje significativo en el tiempo para transformar ambientes $\mathrm{y}$ personalidad del aprendido

Este método resulta útil a la enseñanza de niños con TDAH, pues parte de la experiencia previa y en la práctica de sus conceptos, sin la monotonía de las teorías, estos niños tienen tendencia a no seguir las normas por su conducta denominada rebelde y si se desarrolla su imaginación, tendrán una atención específica muy difícil de lograr en esta población. Los niños con TDAH, pueden proponer, en base a su experiencia mejorar la conectividad-su afectosociabilidad que justo lo que el objetivo busca. La guía del tutor juega un papel representativo del control de las emociones (Mora, Laureano, y Velasco, 2011).

\section{CONCLUSIONES}

Se logró alcanzar el objetivo de la investigación que dio como resultado la relación significativa entre las actitudes emocionales en los niños y su respuesta frente a la escolaridad, evidenciado en su reporte de calificaciones, y sobre todo en el índice de correlación mediante el método estadístico seleccionado.

Las emociones vinculadas a la educación cumplen un rol principal dado que son un motor que incentiva la enseñanza y sobre todo el interés por aprender de cada una de las asignaturas. En el caso de las ciencias naturales existe una mayor respuesta por parte de los alumnos, lo cual es un claro indicador de un posible problema en el desarrollo pedagógico de dicha asignatura.

Es importante establecer con los resultados obtenidos, pautas de mejora en atención a las necesidades detectadas de los estudiantes y de esta forma poder subsanar las deficiencias y que el curso de evolución de su educación mejore. De esta forma su atención en general se mantiene a la expectativa de los nuevos conocimientos y de la forma de transmitirlos, cabe recalcar que, para el diseño de este tipo de programas, se debe incluir otros profesionales como el departamento DECE, quienes, desde la óptica psicológica, pueden contribuir a correcto establecimiento de las pautas o estrategias para los niños ya que su punto de especialización está dirigido a las emociones que ellos sientan. 


\section{REFERENCIAS}

Ángulo, R., y Albarracín , Á. (2018). Validez y confiabilidad de la escala rasgo de metaconocimiento emocional (TMMS-24) en profesores universitarios. Revista Lebret 10, 61-72

Acero, J. (2013). Emoción como exploración. Universitas Philosophica, 26 (52), 133-162.

Aires, L., Teixeira, A., Azecedo, J., Gaspar, M., y Silva, S. (2006). Alteridad y emociones en las comunidades virtuales de aprendizaje Teoría de la Educación. Educación y Cultura en la Sociedad de la Información, $7(2), 74-91$

Alfaro, F. (2012). Temas Controversiales en el TDAH. Revista Latinoamericana de Bioética, 12(2), 100-105

Bermeo Moreno, J., Pérez-García, A., Caballero, R., Antonio, J., Sanjuan Suarez, P., y Rueda Laffond, B. (2012). Psicologia de la Personalidad. madrid: Universidad Nacional de Educación a Distancia

Blanco, J., de Caso, A., y Navas, G. (2013). Variables personales implicadas en el aprendizaje: ¿motivación y/o emoción? International Journal of Developmental and Educational Psychology, 1(1), 505-512

Borrachero, A., Dávila, M., Brígido , M., Gómez, R., y Mellado, L. (2014). Las emociones que experimentaban los futuros profesores de secundaria en el aprendizaje de las ciencias. International Journal of Developmental and Educational Psychology, 4(1), 271-279

Bravo, C. (2018). Como estimular la creatividad en educación. International Journal of Developmental and Educational Psychology, 2(1), 89-97

Burrola, J., Burrola, L., y Viramontes, E. (2016). Inteligencia emocional $\mathrm{e}$ integración grupal en el aula: dos consideraciones en la formación docente. Ra Ximhai, 12(6), 65-176.

Bustillo, M., y Servera, M. (2015). Análisis del patrón de rendimiento de una muestra de niños con TDAH en el WISC-IV. Revista de Psicología Clínica con Niños y Adolescentes 2(2), 121-128
Fernández , J., Del Caño , M., Palazuelo, M., y Marugán, M. (2011). TDAH. Programa de intervención educativa. International Journal of Developmental and Educational Psychology, 1(1), 621-628

González, R., Bakker, L., y Rubiales, J. (2014). Estilos parentales en niños y niñas con TDAH. Revista Latinoamericana de Ciencias Sociales, Niñez y Juventud, 12(1), 141-158

Kolb, D. (1984). Experiential Learning. New Yersey, Estados Unidos: Iwo.

Lolas, F. (2015). Las Emociones. Revista Chilena de Neuropsiquiatría, 46(2), 152.

Luzuriaga, R. (2018). Retos y herramientas generales para el desarrollo de la inteligencia emocional en las aulas. Facultad de Psicología BUAP, México, Sistema Nacional de Investigadores (SNI), México

Marcos, J. (2019). Análisis de las relaciones emociones-aprendizaje de maestros en formación inicial con una práctica activa de Biología. Revista Eureka sobre Enseñanza y Divulgación de las Ciencias 16(1), 34-47

Mora, M., Laureano, A., y Velasco, P. (2011). Estructura de las emociones dentro de un proceso de enseñanza-aprendizaje. Perfiles Educativos, 33(131), 64-79

Orozco, N., y Zuluaga, J. (2015). Teoría de la mente en niños y niñas con trastorno por Déficit de Atención con Hiperactividad "TDAH". Tesis Psicológica, 10(2), 134-148

Ozdemir, S. (2010). Comparación de perfiles de conducta problemática en niños turcos con TDAH y niños sin TDAH. Electronic Journal of Research in Educational Psychology, 8(1), 281-298

Reche, C. (2016). La esgrima extraescolar como propuesta didáctica en TDAH. Cultura, Ciencia y Deporte, 11(33), 217224

Santurde, E., y Del Barrio , J. (2013). TDAH: intervenir en el fomento del apego. International Journal of Developmental and Educational Psychology, 1(1), 265-270 
Scandar, M. (2013). Relación entre los síntomas de TDAH y el aprendizaje escolar en niños preescolares argentinos. Revista Neuropsicologia Latinoamericana, 5(2), 11-23

Tacca Huamán, D. (2010). La Enseñanza de las Ciencias Naturales en la Educación Básica. Investigación Educativa, 146-147
Vásquez, F. (2017). Inteligencia Emocional En Las Organizaciones

Eduacativas. Psicogente, 10(17), 42-59

Yadira, A. (2012). música y aprendizaje significativo. Educere, 13(44), 67-73 